\section{Swedish sports medicine is alive and well!}

\author{
Mats Börjesson, ${ }^{1,2}$ Jon Karlsson ${ }^{3}$
}

The Swedish Society of Exercise and Sports Medicine (SFAIM) brings some of the best Swedish sports medicine and exercise related research to BJSM.

\section{WHAT MIGHT MY TELOMERES HAVE TO DO WITH EXERCISE?}

Sjögren et al (see page 1407) report an intervention study on the effects of physical activity on telomere length. This study demonstrates that reduced sedentary time (but interestingly not physical exercise) was associated with the lengthening of telomeres in white blood cells in older, overweight sedentary people. This emphasises the importance of reducing sedentary time, as well as increasing everyday physical activity. Clinically, these findings reinforce that the first step for overweight patients is to reduce sedentary time.

\section{THE ETHICS AND EFFECTS OF CONCUSSION}

Concussion and the potential for brain injury are increasingly recognised. Parents may be reluctant to allow their children to participate in concussion-prone sports, such as ice-hockey and American football. If a person is concussed, one question is do medical personnel follow the concussion rules? Possibly more than 1000000 people watched the recent World Cup match where a player who sustained a concussion early in the match played on for several minutes. After the game, the player reportedly said, "I did not know where I was."

In the editorial, we discuss some of the important and often very difficult ethical dilemmas facing the team physician in sports. These are probably at least partly caused by the increasing pressure on team physicians due to the high financial stakes, media exposure and prestige in elite sports (see page 1398). We believe these issues need more focus and that education about sports medicine ethics should be included in sports medicine physicians' training and the training of others responsible for the health of athletes. Ethical considerations include practical responses to confidentiality dilem-

\footnotetext{
${ }^{1}$ Swedish School of Sport and Health Sciences, Stockholm, Sweden; '2Department of Cardiology, Karolinska University Hospital; ${ }^{3}$ Department of Orthopedics, Sahlgrenska University Hospital
}

Correspondence to Professor Mats Börjesson, Swedish School of Sport and Health Sciences, Stockholm 114 86, Sweden; mats.brjesson@telia.com mas in elite sports medicine described by Malcolm et al (see page 1410).

Importantly, we know too little about the short-term and long-term sequels after concussion. The paper by Nordström et al (see page 1447) highlights the chance of injury after concussion. This paper investigated 47 elite soccer teams in 10 European countries over 11 seasons. Two different survival models analysed whether concussion increased the risk of subsequent injury during the first year after concussion. Compared with the risk following other injuries, concussion was associated with a significantly increased risk (56\%) of a second injury during the first year, even after adjustment for the number of injuries in the year preceding the concussion. This number is high and measures such as in-depth neurological and cognitive assessment are needed before return to play. We know too little about safe return to play after concussion. Gradual return to play is usually recommended at 7-10 days after concussion, but do we really know whether this is enough? Symptoms are often subtle and that is why it is valuable to compare preseason and postconcussion tests, similar to other established preseason tests, in all players.

\section{SWEDES ARE GREAT AT TENDON RESEARCH!}

We often talk about tendinosis, although we really do not know where the pain comes from and how structural tissue changes are related to tendon pain. Backman et al (see page 1414) have shown that tumour necrosis factor- $\alpha$ can induce programmed cell death in human tenocytes in vitro. Importantly, substance $\mathrm{P}$ is also upregulated in tenocytes as a response to stress, which can then mediate against cell death and might play a role in the development of tendinosis. An increased in-depth understanding of this effect and of the role of mediators might move us closer to the understanding of tendon pain and tendon repair.

The changes in sensation in people with tendinopathy were reviewed by Heales et al (see page 1400), this review showed that there were bilateral deficits in sensation in people with unilateral tendinopathy. Interestingly, training can have a bilateral effect through the central nervous system and Alfredson et al reports that unilateral surgical treatment in people with mid-portion Achilles tendinopathy may result in bilateral recovery. The authors recommend unilateral surgery and then a 'wait-and-see' strategy if the contralateral side is painful as well.

\section{WHAT ABOUT PREVENTION?}

Injury prevention is important in all sports as it is better to prevent than treat. A paper by Lindblom et al (see page 1425) completed a 3-year follow-up of a successful randomised trial in Sweden and examined the implementation of the programme among participants and coaches. The study showed that implementation of a neuromuscular training programme in female adolescent football players can significantly reduce the subsequent number of injuries.

Finally, a study from an award-winning $\mathrm{PhD}$ thesis in orthopaedics in Sweden in 2012 is reported. Björnsson Hallgren et al (see page 1431) randomly allocated 100 people with subacromial pain (often called the impingement syndrome) to either an exercise group or a control group. The exercise strategy that included range of motion, strength and endurance significantly reduced the need for surgery in these patients. Surprisingly, less than $50 \%$ of patients with disabling subacromial pain needed surgery. Only 24\% of patients in the exercise group decided to undergo surgery, compared with $63 \%$ in the control group. They also showed that full thickness rotator cuff tear and low preoperative functional score (Constant score) were predictors of poorer outcome.

\section{SFAIM CONFERENCE-MAY 2015!}

We close this SFAIM BJSM issue warm-up with a reminder to hold 7-9 May 2015 free for the Swedish Society of Physical Activity and Sports Medicine Annual meeting in Linkoping. Get your abstracts ready, share your ideas with the organising committee (Martin Hägglund martin.hagglund@liu.se). Encourage your colleagues who are not yet members of SFAIM to join (www.svenskidrottsmedicin.se)! They will be joining a motivated, fun and hardworking society that is doing important work. Great value and includes full access to BJSM!

\section{Competing interests None.}

Provenance and peer review Not commissioned; internally peer reviewed.

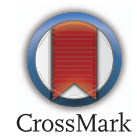

To cite Börjesson M, Karlsson J. Br J Sports Med 2014;48:1397.

Accepted 31 July 2014

Br J Sports Med 2014;48:1397.

doi:10.1136/bjsports-2014-094099 